\title{
UT1 and relativistic theory of Earth rotation
}

\author{
S. A. Klioner
}

Lohrmann Observatory, Dresden Technical University, 01062 Dresden, Germany

\begin{abstract}
The influence of post-Newtonian effects in the rotation of Earth on UT1 are investigated and found to be negligible.
\end{abstract}

Keywords. relativity, time, reference systems

Using the numerical code for the post-Newtonian theory of Earth rotation (Klioner et al. (2008), Klioner et al. (2009)) it is not difficult to investigate the influence of the relativistic effects on the angular velocity of the Earth that is directly related to UT1. Two numerical integrations for the whole validity range of DE403 has been performed. First integration was purely Newtonian (all relativistic effects have been switched off). For the second integration several dynamical relativistic corrections were switched on: (1) full post-Newtonian torque was used; (2) relativistic time transformation between TT (argument of integration) and TDB (argument of the ephemeris) were applied; (3) relativistic scaling of parameters were applied. Using the results of both integrations, the angular velocity of proper rotation of the Earth was computed. The difference of the angular velocities obtained in two integration is shown on Fig. 1. One can see that, contrarily to claims of some authors, the influence of relativity on the LOD and therefore on UT1 is fully negligible and remains well below $1 \mu \mathrm{s}$. The effects of geodetic precession, being of kinematical nature, are another potential source of the variation of the LOD and will be discussed elsewhere.

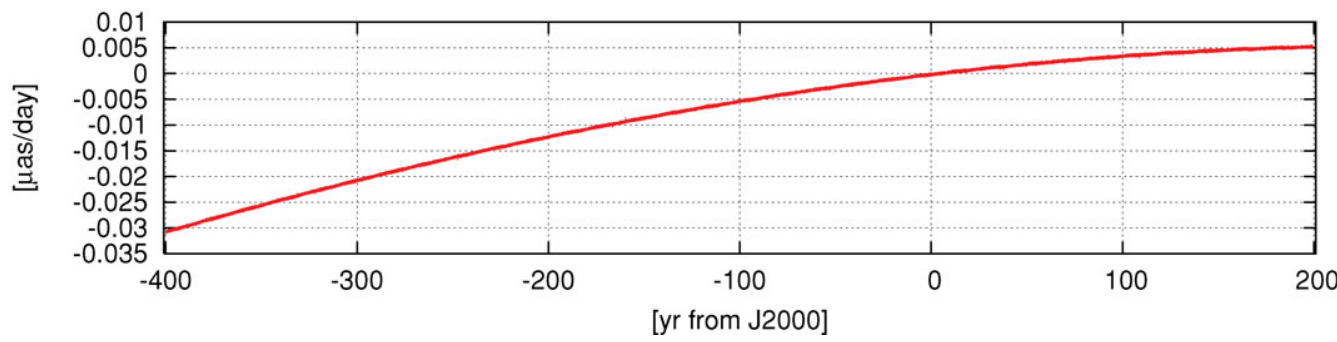

Figure 1. Difference between angular velocities of the Earth obtained in fully post-Newtonian model and in purely Newtonian one.

\section{References}

Klioner, S. A., Soffel, M., \& Le Poncin-Lafitte, Chr., 2008, "Towards the relativistic theory of precession and nutation", In: The Celestial Reference Frame for the Future (Proc. of Journées 2007), N. Capitaine (ed.), Paris Observatory, Paris, 139

Klioner, S. A., Gerlach, E., \& Soffel, M., 2009, Relativistic aspects of rotational motion of celestial bodies, In: Relativity in Fundamental Astronomy, Proc. of the IAU Symposium 261, S. Klioner, K. Seidelmann, M. Soffel (eds.) Cambridge University Press, Cambridge, in press 